\title{
D'une restauration hydraulique et écologique à un territoire de projet : le cas du Haut-Rhône français
}

\author{
André Micoud \\ Sociologue, Université Jean Monnet, Centre Max-Weber, UMR 5283, 42023 Saint-Étienne, France
}

\begin{abstract}
À partir d'une analyse empirique fine d'un terrain rhodanien, l'auteur ausculte les rapports qui se nouent entre sciences, engagement et politique pour aboutir à l'invention d'un territoire. Il montre comment des responsables locaux en sont venus à considérer le Rhône non plus comme une frontière mais comme l'épine dorsale d'un territoire à l'issue de plusieurs décennies $\mathrm{d}^{\prime}$ investissement à la fois cognitif et militant autour de ce fleuve. Le territoire apparaît ici non pas comme réceptacle passif d'actions publiques, ni comme simple objet de luttes politiques. Il est abordé comme un processus qui s'inscrit dans des limites spatiales et temporelles bien plus larges que celles fixées par la communauté qui le porte, et qui se nourrit de multiples et complexes interactions : entre transformations physiques du milieu, idéalités spatiales, régulations, gouvernance...
\end{abstract}

La Rédaction

\section{Mots-clés :}

recherche ; territoire ; fleuve ; restauration ; gouvernance ; Haut-Rhône

\section{Keywords:}

research; river; restoration; governance; French Upper-Rhone
Résumé - Alors que les aménagements réalisés par la Compagnie nationale du Rhône étaient censés les en protéger, des communes riveraines du fleuve Rhône ont subi une inondation en 1990. Aujourd'hui réunies dans un Syndicat du Haut-Rhône devenu porteur d'un territoire de développement très actif, elles font de cette catastrophe naturelle le point de départ de l'histoire de ce syndicat. La recherche a montré que cette version officielle de l'histoire ignorait l'essentiel, à savoir que, depuis des décennies, ce tronçon du fleuve était l'objet d'un investissement cognitif considérable de la part des scientifiques de l'Université de Lyon, jusqu'à ce que, relayés par des experts-militants, ils parviennent à le faire choisir comme terrain d'expérimentation d'un programme de restauration écologique et hydraulique. C'est en se saisissant de ce programme que le Syndicat du Haut-Rhône a su se transformer en un acteur du développement.

\begin{abstract}
From a scheme of ecological and hydraulic restoration to the formation of a political force: the case of the Upper-Rhone in France. An ecological and hydraulic restoration programme was launched in 2001 on a section of the French Rhone upstream of Lyon. Two research teams, one specializing in life sciences, the other in social and human sciences (SHS), were charged with assessing the effects of the programme to highlight the changes that occurred in the relations between the rivers and the human collectives living along them. This article reports on the results of the SHS team based on a dual approach - consideration of the local situation and of the programme chronology. The research showed rapidly that the main effect of the programme had been the establishment in 2003 of a Syndicate of the Upper-Rhone, which become the leading actor of a development centring around the river for the waterside municipalities situated on the borders of the three departments concerned. But the main reason underlying the location of the programme on this section of the river was that for decades this section had been the target of considerable cognitive investment by scientists from Lyon University. The arguments developed, relayed by expert activist ecologists, were able to convince the authorities to select it as one of the experimental sites for a new sustainable management of rivers. By uniting the municipalities around this programme, the Upper-Rhone Syndicate established itself as a valid interlocutor to cooperate in this management.
\end{abstract}

\footnotetext{
Auteur correspondant : andre.micoud@sfr.fr

André Micoud est directeur de recherche honoraire du CNRS et il était président de la Maison du fleuve Rhône, association dédiée à la connaissance anthropologique des fleuves créée en 1988 et dissoute en 2014.
} 
L'article que l'on va lire résulte d'un travail mené dans le cadre d'un programme national de recherche lancé en 2007 à l'instigation du ministère de l'Écologie et du Développement durable, du Cemagref ${ }^{1}$ et du CNRS. Intitulé «Eaux et territoires », ce programme se proposait de réunir des spécialistes des sciences de la vie et de la Terre et des sciences sociales pour mieux comprendre les transformations des relations entre les fleuves et les rivières et les collectifs humains qui habitent leurs rives ${ }^{2}$.

En répondant conjointement à cet appel à recherche, la Maison du fleuve Rhône, d'un côté, et le Laboratoire d'écologie des hydrosystèmes fluviaux $\left(\mathrm{LEHF}, \mathrm{UMR} 5023^{3}\right.$ ), de l'autre, se proposaient d'essayer de mieux comprendre comment une action de restauration hydraulique et écologique sur une portion du Rhône avait pu participer à la structuration d'un territoire de «projet » aujourd'hui géré par le Syndicat du HautRhône. Les deux institutions de recherche ont en effet formulé l'hypothèse que l'histoire du territoire ainsi appelé " Haut-Rhône », du nom du syndicat constitué en avril 2003, pouvait constituer un cas exemplaire des intrications complexes amenant un collectif territorialisé à se doter d'un instrument adéquat pour organiser son avenir autour d'un fleuve (voir Carte).

Associées au suivi de ce programme de restauration, sur le versant de ses effets écologiques pour le LEHF et sur le versant des effets socioéconomiques pour la Maison du fleuve Rhône, les deux institutions estimaient qu'un retour réflexif sur une expérience partagée était nécessairement porteur de connaissances nouvelles.

Implicitement donc, un "terrain » était choisi, à la fois délimité spatialement et découpé chronologiquement. L'histoire de la recherche, qui est aussi en partie son résultat, est celle de la remise en cause progressive de ce présupposé ethnographique selon lequel tous les déterminants d'une histoire locale se tiendraient

1 Centre national du machinisme agricole, du génie rural, des eaux et des forêts, devenu Institut de recherche en sciences et technologies pour l'environnement et l'agriculture (Irstea) depuis février 2012.

2 Le rapport issu de cette recherche Le Haut-Rhône français : d'unfleuverestauréà la construction d'un territoire (Maison du fleuve Rhône, LEHNA, Syndicat du Haut-Rhône, septembre 2011, http:/ / temis.documentation.developpement-durable.gouv.fr/ documents/Temis/0081/Temis-0081083/21633_rapport.pdf) a été rédigé par André Micoud, Gilles Armani et Jean-Michel Olivier avec la participation de Yohann Desbois et Olivier Pignet. Je remercie tout particulièrement Gilles Armani qui a réalisé la plus grande partie des entretiens de terrains. Je remercie également Christelle Morel-Journel pour sa relecture vigilante de cet article.

3 Aujourd'hui le Laboratoire d'écologie des hydrosystèmes naturels anthropisés (LEHNA), qui a pour tutelle le CNRS, l'Université Lyon 1, l'École nationale des travaux publics d'État et l'Inra. enfermés dans les limites spatiales qu'habite une communauté, ainsi que dans les origines temporelles qu'elle donne à son histoire.

Autrement dit, il est apparu peu à peu qu'il n'était pas possible d'expliquer comment un territoire comme celui du Syndicat du Haut-Rhône avait pu advenir si l'on s'en tenait à ce seul tronçon du fleuve et à la seule histoire officielle qu'il tenait sur lui-même ${ }^{4}$. Si, comme l'a écrit Béatrice Frankael (1992), « l'institution tient son pouvoir de sa capacité à effacer les traces de son écriture », il nous appartenait, pour pouvoir comprendre sa naissance, de nous affranchir de ce cadrage. Mais qu'on ne se méprenne pas sur nos intentions : il n'était nullement question pour nous d'écrire une histoire «critique» se donnant pour mission de mettre au jour une "vérité » que ses acteurs auraient voulu escamoter. Notre propos est beaucoup plus modeste, et consiste plutôt à exposer ce à quoi notre pratique de chercheurs en sciences humaines nous oblige, à savoir de rendre compte de la façon la plus exhaustive possible de la multiplicité des réseaux qui - nous incluant nous-mêmes, LEHF et Maison du fleuve Rhône - avait pu concourir à cette intrication singulière entre des hommes et un fleuve.

Certes, une crue a bel et bien eu lieu en février 1990, comme le dit l'histoire officielle de la naissance du Syndicat du Haut-Rhône. Sauf que tout le problème réside dans la question de savoir pourquoi, à cet événement naturel, les responsables locaux ont réagi de la façon dont ils l'ont fait, qui les a amenés au final à inventer un nouveau territoire organisé autour du fleuve. La cause principale qui nous paraît rendre raison de cette attitude est la suivante : c'est parce que le Haut-Rhône faisait l'objet d'un double investissement, à la fois cognitif et militant depuis de nombreuses années que, plutôt que de réclamer une défense immédiate contre les crues, les responsables locaux ont été amenés à prendre le temps de mieux connaître le fonctionnement du fleuve et que, forts de ce recul, ils en sont venus à reconnaître que leur destin lui était lié.

Si notre programme de recherche consistait bien en une analyse rétrospective de la manière dont la « restauration hydraulique et écologique » du Haut-Rhône initiée en 2001 avait pu participer à la construction de cette section du fleuve comme un «territoire » de projet, la chronologie des investissements cognitifs et militants dont cette portion du fleuve a été l'objet exigeait toutefois de remonter bien avant dans le temps et de s'éloigner

\footnotetext{
4 «Le Rhône, un fleuve fédérateur : de la crue au projet de territoire » : c'est sous ce titre (d'un exposé de la présidente du Syndicat du Haut-Rhône au colloque «Le patrimoine, un avenir pour les territoires ", Centre culturel français, Cluj, Roumanie, 26-28 mai 2011) qu'on a pu confirmer l'hypothèse qu'il existe bien une histoire officielle du territoire du Syndicat du Rhône, qui la fait démarrer à partir de la crue de 1990.
} 


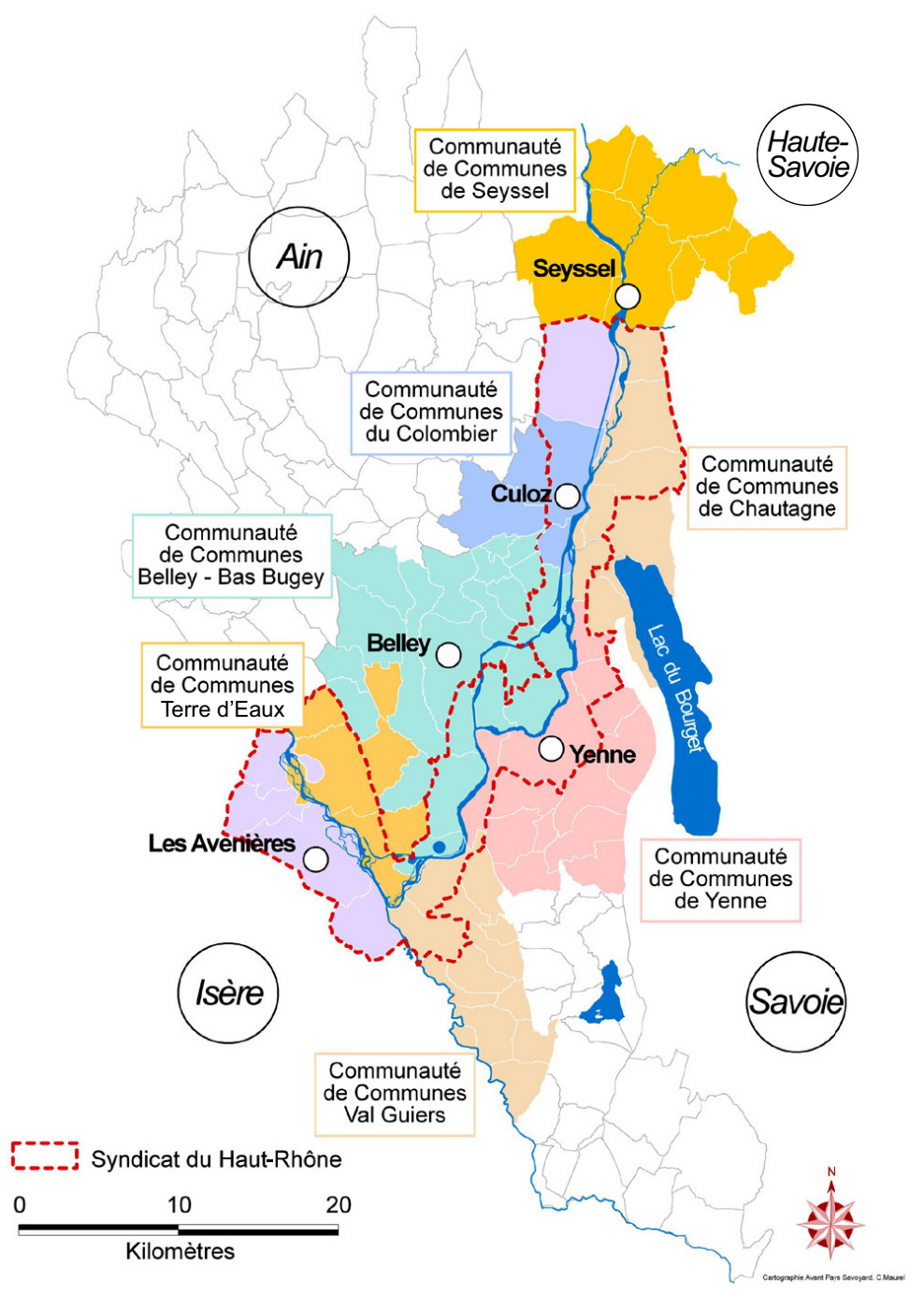

Carte. Périmètre du Syndicat du Haut-Rhône (source : Micoud et al., 2011).

aussi du seul terrain du Haut-Rhône. D'autres lieux d'investigation s'imposaient : un laboratoire CNRS de l'Université de Lyon 1 dans les années 1970, la commune de Vernaison en aval du barrage-écluse de Pierre-Bénite au sud de Lyon en 1985, le siège de la Frapna ${ }^{5}$ sur le campus de la Doua à Villeurbanne, le ministère de l'Environnement, l'agence de l'eau à Lyon, etc.

\section{Une terre des confins}

Précisons tout d'abord que parler du Haut-Rhône est une expression que les Suisses utilisent pour désigner le Rhône valaisan, alors qu'en France, ce terme est employé par la Compagnie nationale du Rhône (CNR) dans le découpage qu'elle opère à partir de 1933 pour mener à bien l'aménagement du fleuve qui lui a été concédé.

5 Fédération Rhône-Alpes des associations de protection de la nature.
Dans ce cadre, le Haut-Rhône désigne le fleuve à l'amont de Lyon. C'est sur cette portion qu'a été érigé, en 1948, le premier grand aménagement avec le barrage de Génissiat affecté à la production d'hydroélectricité ${ }^{6}$. Nous noterons qu'en aucun cas cette appellation ne renvoie à un "territoire ", il ne s'agit que d'un découpage technique posé par des hydrauliciens.

Dans cette portion de son cours, le Rhône est un fleuve rural, puissant et turbulent, mais qui renforce surtout la frontière qui délimitait hier des empires, puis des pays, et aujourd'hui des départements. Situé aux confins des départements de 1'Ain, de l'Isère et de la Savoie, le fleuve n'est pas repéré localement comme un élément majeur du territoire ni même porteur de

\footnotetext{
6 Seul barrage français coupant le fleuve, le barrage de Génissiat a noyé le site exceptionnel et touristique qu'étaient les «Pertes du Rhône».
} 
potentialités (Monneret, 2001). Les activités touristiques des trois départements qu'il délimite sont principalement tournées vers les lacs et les montagnes, éléments caractéristiques de la région qui bénéficie d'une excellente image: verdure, plein air, propreté des eaux (avec les campagnes d'épuration des lacs du Bourget et d'Annecy). En bref, un fleuve qui sépare des espaces ruraux, chacun tourné vers sa préfecture respective dont l'éloignement leur laisse peu espérer d'en être entendus un jour.

Enfin, durant les années 1970-1980, le Rhône qui traverse cette région est surtout un fleuve peu aménagé sur lequel il est possible d'observer des fonctionnements hydromorphologiques et hydrobiologiques proches des conditions «naturelles », bien que parfois perturbés par les barrages en amont. En effet, les quatre autres aménagements sur cette portion (avec dérivation et usine hydroélectrique à faible hauteur) n'ont été achevés qu'entre 1980 et 1986. Un cinquième prévu à Loyettes à la confluence avec l'Ain ne sera jamais réalisé, suite à une contestation emmenée par la Frapna (Michelot, 1990).

Et c'est bien ce caractère « naturel » qui va être à l'origine du double investissement qu'on a dit scientifique et militant à la fois : un tronçon du Rhône qu'il est possible d'étudier comme un modèle de grand fleuve, parce que non perturbé par les activités humaines, et un tronçon du Rhône qu'il faut protéger, parce que le dernier à n'avoir pas encore été aménagé. Nous le verrons plus loin, ces deux types d'investissements que l'on pose ici comme séparés vont se révéler en fait fortement liés. Ce sont eux, en effet, qui vont se trouver mobilisés comme ressource pour faire émerger ce territoire de projet (Lajarge et Roux, 2007).

Comme on vient de le voir, cette terre des confins - hormis son appellation géomorphique et technicienne, qui ne désigne que le fleuve qui la traverse - n'a pas d'identité propre. Comme tout espace, il est déjà découpé administrativement, mais en l'occurrence de telle sorte que les parties qui le composent se trouvent chacune indexée à des autorités périphériques départementales différentes. C'est sur la base de cet existant que ce que l'on appelle un «territoire de projet » peut apparaître quand une autorité propose d'instaurer un nouveau découpage au motif qu'il serait plus adéquat pour des perspectives nouvelles. Il y a eu ces dernières décennies un intense travail de recherche et de réflexion autour de cette notion de territoire, travail auquel nous avons contribué (Micoud, 1995, 2000 ; Fourny et Micoud, 2002). Par contre, il ne nous a pas semblé possible, au risque de trop alourdir le propos, de résumer les apports de ces approches. Le lecteur pourra s'il le souhaite se reporter au numéro de la revue Ethnologie française consacré au thème "Territoires en question » $(2004,1)$ ainsi qu'à Thierry Paquot (2011) pour avoir une vue d'ensemble sur cette notion. Plus spécifiquement, sur les rapports $\mathrm{du}$ «territoire de projet » à son indispensable narration, on pourra se référer à Dominique Pagès (2010), de même que, sur le rôle que peut avoir un événement dans le processus de son émergence, on lira avec profit l'ouvrage de Julien Langumier (2008). En bref, si l'on retient la définition selon laquelle un territoire est un espace en tant qu'une autorité politique s'en occupe, en commençant par le circonscrire et le nommer, la description qui nous occupera ici est celle de l'émergence d'un territoire inédit rassemblant autour d'une nouvelle sémantique du fleuve des espaces qu'on considérait jusqu'alors comme simplement riverains.

\section{Le Haut-Rhône, un laboratoire pour les sciences de la vie et de la Terre...}

Un Haut-Rhône peu aménagé certes, mais c'est pourtant bien à cause des tout premiers aménagements qu'il va commencer à être étudié par les scientifiques. Les premiers travaux consistent en des inventaires piscicoles réalisés par des professeurs de biologie de l'Université de Grenoble et des ingénieurs du génie rural et des eaux et forêts (Carrel, 2002). Ils font suite à la construction des barrages sur cette portion; le barrage franco-suisse de Chancy-Pougny construit dans les années 1920, celui de Verbois mis en service en Suisse en aval de Genève en 1943, et enfin celui de Génissiat inauguré en 1948.

Après ces premières transformations, c'est surtout grâce à la perspective de la finalisation de l'aménagement du fleuve par la CNR suite à la crise énergétique de 1973 que ce secteur rhodanien va constituer un véritable laboratoire pour les scientifiques. Durant toute cette période, les savoirs s'accumulent. Et c'est sous l'égide d'un professeur d'hydrobiologie de l'Université Lyon 1, Albert-Louis Roux ${ }^{7}$, que, dans les années 1975-1976, commencent ces recherches au sein du Laboratoire d'écologie des hydrosystèmes fluviaux (le LEHF déjà nommé). Un article de Jacques Juget publié en 1976 dans une revue minuscule peu connue à l'époque, le Bulletin écologique, est le premier à porter l'attention sur les eaux souterraines accompagnant l'écoulement du fleuve (Juget et al., 1976). En 1978, la thèse de Jean-François Perrin sur les macro-invertébrés benthiques du Rhône «vif » constitue une très bonne illustration du travail entrepris et des difficultés rencontrées.

En effet, ces premières investigations sur le Rhône mettent rapidement en évidence à la fois la complexité du milieu étudié et les difficultés méthodologiques associées à l'échantillonnage dans ce système de grandes dimensions. Toutefois, malgré les difficultés d'accès aux données de terrain, le professeur Roux et ses collaborateurs, s'appuyant sur des compétences reconnues en

\footnotetext{
Albert-Louis Roux est actuellement membre del'Académie de l'eau.
} 
systématique et en écologie des organismes aquatiques, font preuve d'une curiosité scientifique et d'une détermination exceptionnelles, mettant en avant un champ thématique de recherche innovant et prometteur.

L'existence de ces travaux précurseurs débouche sur un Piren (Programme interdisciplinaire de recherche sur l'environnement) « Haut-Rhône », mis en œuvre à la fin de l'année 1979 à l'initiative conjointe du CNRS et du ministère de l'Environnement. Ce programme va élargir le nombre de disciplines concernées en incluant des géographes et des spécialistes du milieu souterrain, jusqu'ici surtout impliqués dans l'étude des karsts ${ }^{8}$ et de leur faune. En 1982, les recherches accomplies s'inscrivent dans une publication de CNRS Éditions sous un titre, quasi identique à celui du Piren (Propositions pour une méthodologie cartographique polythématique appliquée à la gestion écologique des eaux. Étude d'un fleuve dans sa vallée : le Haut-Rhône français) (Piren, 1982). L'ensemble des systèmes aquatiques, semi-aquatiques et terrestres liés au fleuve sont alors pris en compte, donnant naissance au concept d'hydrosystème et faisant émerger l'idée des quatre dimensions du fleuve : longitudinale, latérale (connectivité avec les milieux annexes), verticale (connectivité avec le milieu hyporhéique ${ }^{9}$ ) et temporelle (la prise en compte de la dynamique de ces systèmes est à la base des concepts développés) (Amoros et Bravard, 1985). Des articles scientifiques sont publiés en nombre dans des revues internationales. Par conséquent, quand les travaux d'aménagement du Haut-Rhône sont terminés en 1986, le Haut-Rhône français est connu de tous les spécialistes mondiaux de l'écologie des fleuves. C'est donc à partir des recherches effectuées sur cette portion du fleuve que seront élaborés les principaux concepts structurants d'hydrosystème à quatre dimensions, d'unité fonctionnelle, de séquences évolutives, ainsi que celle, plus normative, «d'espace de liberté ». Cette dernière notion va connaître une rapide fortune puisqu'elle sera reprise dans une plaquette subventionnée et diffusée par le ministère de l'Environnement en 1991 Fleuves, sources de vie ${ }^{10}$, avant $\mathrm{d}^{\prime}$ entrer dans la réglementation sous la forme d'une mention dans le Schéma directeur

\footnotetext{
8 Structures géomorphiques faites de cavités souterraines produites par érosion hydrochimique dans les roches carbonatées, principalement les calcaires.

9 Zone poreuse formée par les sédiments de fond dans laquelle se mélangent les eaux souterraines et de surface.

10 Sous la plume de Monique Coulet, à l'époque chargée de recherche au CNRS, au sein du Laboratoire d'écologie des eaux douces de l'Université de Lyon 1, URA 1451. Outre le ministère de l'Environnement, ont contribué à la publication de cette plaquette, le Conseil supérieur de la pêche, le Bureau de la convention Ramsar et... la Frapna dont Monique Coulet est alors la présidente. Cela nous permet de constater la grande porosité entre les milieux de l'Administration et ceux des réseaux militants de la défense de l'environnement (Charvolin, 2003).
}

d'aménagement et de gestion des eaux (SDAGE) Rhône-Corse-Méditerranée de 1994.

Le second Piren, «Vallées fluviales », programme pluridisciplinaire par principe, est l'endroit où naissent des collaborations fructueuses associant des chercheurs de différentes disciplines. Comme Jean-Paul Bravard qui rédige sa thèse de $3^{\mathrm{e}}$ cycle en géographie et en géomorphologie sur les marais de Chautagne (1981), puis sa thèse de doctorat d'État sur le Haut-Rhône (1985) pour devenir ensuite un des spécialistes mondiaux des fleuves, ce qui lui vaut la médaille d'argent du CNRS en 2004. Mentionnons aussi Claude Amoros, spécialiste des cladocères ${ }^{11}$ et des annexes fluviales, puis de l'écologie des macrophytes aquatiques, ce qui lui permet de réaliser à la demande de la CNR un atlas des lônes ${ }^{12} \mathrm{du}$ Rhône de la frontière suisse à la Camargue, avant de prendre la direction du LEHF. Et il faudrait encore citer les travaux de Guy Pautou et Jacky Girel, de l'Université Joseph-Fourier à Grenoble, sur les associations végétales terrestres des plaines alluviales, débouchant sur une cartographie de la végétation et aussi ceux du biostatisticien Daniel Chessel, qui jouera un rôle important dans le développement de méthodes spécifiques d'analyse de données.

Dans cette dynamique, le Piren « Haut-Rhône », puis le Piren « Vallées fluviales » offrent un cadre pour la réalisation de nombreux travaux de thèse de doctorat. Afin d'allier au mieux recherche fondamentale et recherche appliquée, et pour assurer une meilleure gestion administrative et financière des contrats (notamment ceux obtenus de la CNR pour les suivis hydrobiologiques consécutifs aux aménagements hydroélectriques récemment construits de Chautagne, Belley et BrégnierCordon), A.-L. Roux crée une association (loi de 1901), l'Association Rhône-Alpes pour l'étude des problèmes biologiques de la pêche (ARALEPBP). Cette structure permet de financer plusieurs thèses et contrats postdoctoraux à une période où ce type d'allocations était plutôt rare. La plupart des étudiants de cette époque (une vingtaine) travaillent aujourd'hui encore dans le domaine de l'écologie fluviale dans différents laboratoires en France ou à l'étranger.

À la fin des années 1980, la dynamique scientifique de ce domaine commence toutefois à s'essouffler au laboratoire de l'Université Lyon 1. Pour capitaliser ses résultats, A.-L. Roux lance la rédaction d'un ouvrage collectif qui parait sous la signature de Claude Amoros et de Geoffrey E. Petts en 1993. Il est intitulé Hydrosystèmes fluviaux et une édition en anglais sort en 1996. Au début des années 1990, le laboratoire recrute un chercheur de

\footnotetext{
11 Petits crustacés aquatiques appelés également puces d'eau.

12 Bras secondaire d'un fleuve aux eaux stagnantes en dehors des périodes de crues.
} 
renommée internationale, Bernhard Statzner (diplômé des universités de Kiel et de Karlsruhe) à qui A.-L. Roux confie la coordination et l'édition d'un numéro fort important de la revue Freshwater Biology (Statzner et al., 1994) faisant la synthèse des travaux réalisés sur le Rhône et portant sur le test du concept d' « habitat templet » (modèle de répartition des habitats des espèces). Ce numéro spécial connaît un succès mondial.

\section{Un laboratoire très présent sur le terrain}

Si toutes ces recherches donnent lieu à des conceptualisations qui trouvent leurs débouchés dans des revues scientifiques internationales, elles impliquent en même temps une grande proximité avec le terrain, du fleuve certes, mais aussi de tous ses gestionnaires. Une proximité qui se manifeste, par exemple, par le fait que A.-L. Roux devient à cette époque le président du Conseil scientifique du comité de bassin RhôneMéditerranée-Corse. Une chronique de la recherche qui ne s'en tiendrait qu'à la seule dimension académique serait très insuffisante. Comme l'a montré Michel Callon (1986), les travaux scientifiques ne se réalisent pas seulement en laboratoire, notamment pour les chercheurs en sciences de la vie et de la Terre.

Voilà pourquoi, quand le Syndicat intercommunal de protection des berges et bordures du Rhône de Savoie (SIPBBRS), créé en 1991 suite à la crue de février 1990, commande la réalisation d'études sur l'écologie du fleuve dans le secteur géographique concerné (essentiellement les aménagements de Chautagne, Belley et, en partie, Brégnier-Cordon), il sollicite pour cela Jean-Paul Bravard, éminemment connu sur le terrain, non seulement parce qu'il y a réalisé sa thèse, mais surtout parce qu'il y a noué des liens très forts avec les acteurs de la région. Ce dernier fait appel à Jean-Michel Olivier, en passe d'être intégré au CNRS dans le laboratoire de A.-L. Roux. De cette collaboration sortira une contribution collective incluant un ingénieur de la CNR (Klingeman et al., 1998). En effet, parce que les agents de cette compagnie possèdent toutes les données hydrologiques, de nombreuses données de terrain et une très bonne connaissance des sections aménagées, il était indispensable pour les chercheurs de coopérer avec eux. Outre ces contacts étroits déjà existants avec les agents de la CNR, les chercheurs, soucieux de pouvoir poursuivre leurs recherches et désireux de les faire connaître, nouent aussi de nombreuses relations avec les différents élus des communes concernées.

Toutefois, un bon nombre d'entre eux ne sont pas que des chercheurs, ils sont aussi des citoyens qui militent pour la protection de la nature, ce qui peut expliquer l'importance de leurs investissements. Un autre site à l'aval de ce même tronçon du fleuve, à sa confluence avec l'Ain, a connu lui aussi un fort investissement scien- tifique et militant dans des années antérieures, où l'on retrouve en partie les mêmes acteurs (Michelot, 1990).

\section{Le rôle des experts-militants ${ }^{13}$}

Pourquoi le programme de restauration hydraulique et écologique a-t-il eu ce tronçon du Haut-Rhône comme premier terrain ? Comme la lettre ministérielle de juillet 1998 en porte la trace explicite, il y a un lien entre l'abandon du canal Rhin-Rhône et ce programme de restauration. Pour comprendre la nature de ce lien, il faut encore une fois se transporter sur une autre scène. En juin 1997, suite à la dissolution de l'Assemblée nationale, la « gauche plurielle » accède au pouvoir. Alors qu'en 1996, le Premier ministre Alain Juppé avait lancé une consultation auprès du grand public pour la construction d'un canal entre le Rhin et le Rhône confiée à la CNR, le collectif d'opposants, formé de 200 associations coordonnées par l'organisation Saône \& Doubs Vivants, la Frapna et le WWF, parvient à publiciser les arguments en défaveur d'un projet dénoncé comme "pharaonique ». Le projet nucléaire de Superphénix et celui du canal Rhin-Rhône sont abrogés par le Premier ministre en octobre 1997 et l'on peut penser que Dominique Voynet, alors ministre de l'Environnement et élue de la région Franche-Comté, a compté dans cette décision, tout comme a vraisemblablement pesé l'avis négatif que le Conseil scientifique du Comité de bassin Rhône-Méditerranée-Corse, présidé alors par A.-L. Roux, avait émis en 1995.

Le fait que l'État ait ensuite demandé à la CNR de réinvestir les dépenses prévues dans ce projet avorté dans le programme décennal de restauration hydraulique et écologique du Rhône montre bien que ce dernier constitue une espèce de "compensation ». Selon ses dires, la présidente de la Frapna, Monique Coulet, aurait joué un rôle déterminant dans cette décision au cours d'une rencontre avec D. Voynet en septembre 1998, insistant au passage pour que le site du Haut-Rhône figure dans ce programme. Signalons que M. Coulet a succédé à A.-L. Roux à la présidence de la Frapna quand les rapports de celui-ci avec la CNR sont devenus peu compatibles avec ce poste. Ainsi que le note Carole Barthélémy dans son rapport, empruntant la terminologie de Pierre Muller sur les "référentiels d'action » (Muller, 2003), ce passage d'un projet de grand canal à celui d'une restauration écologique marque la création d'un nouveau référentiel dans lequel un Rhône hydrobiologique succède au Rhône des seuls hydrauliciens (Barthélémy, 2006, p. 24), mutation que l'on doit en

\footnotetext{
13 Sur cette notion, voir Micoud, 2007. On a soutenu dans cet article que, du fait qu'elles ont été les premières à utiliser la science avec le plus de détermination, les mobilisations environnementales ont rendu caduque l'opposition traditionnelle entre le savant et le politique. D'où l'invention de cet oxymore.
} 
grande partie à l'action de ces scientifiques qui furent aussi des « entrepreneurs écologistes ».

Pour comprendre les raisons de cet autre investissement cognitif, il convient là aussi de remonter dans le temps et de se déplacer sur le cours du fleuve. Nous sommes en 1985-1986 et il faut se placer à l'aval immédiat de Lyon, sur le site de Vernaison où, suite à l'aménagement du barrage-écluse de Pierre-Bénite (en 1966), le maire de Vernaison déplore que l'ancien Rhône qui arrose sa commune ne soit plus qu'une petite rivière quand l'essentiel du débit a été détourné sur le canal navigable (sur un débit total de $1000 \mathrm{~m}^{3} / \mathrm{s}, 990$ vont dans le canal et 10 dans le vieux Rhône !). Il souhaite retrouver le fleuve d'antan et s'adresse à la présidente de la Frapna, Monique Coulet, pour soutenir son action. Alors que le projet initial consistait à créer un seuil en aval pour remonter le niveau de l'eau (qui aurait alors été stagnante), M. Coulet, au cours d'une réunion où sont présents tous les décideurs, propose que soit augmenté le débit réservé - ce qui ne peut qu'entraîner une perte de production électrique pour EDF, qui s'y opposera fermement au début. Finalement, avec les appuis de l'Agence de l'eau et du Conseil général du Rhône (sans compter le soutien des « experts » déjà connus que sont J.-P. Bravard et C. Amoros), un arrangement est trouvé grâce à l'installation d'une miniturbine sur le vieux Rhône pour récupérer une partie de l'énergie perdue. La région Rhône-Alpes participera au financement après que Mme Coulet parvint à convaincre les élus régionaux verts de financer un équipement... pour la CNR. Précisons ici, autre illustration de l'implication des " expertsmilitants ", que le groupe des élus verts à la région est présidé par Philippe Lebreton, professeur de physiologie végétale à l'Université Lyon 1 et fondateur de la Frapna en $1971^{14}$.

L'augmentation du débit de 10 à $100 \mathrm{~m}^{3}$ devait permettre de retrouver un "fleuve vif et courant ${ }^{15}$ ", expression qui fait florès et que l'on retrouve dès lors comme un leitmotiv. Les travaux de génie écologique (restauration de 3 annexes fluviales) sont effectués entre 1998 et 2000, l'augmentation de débit réservé étant effective le $1^{\text {er }}$ septembre 2000. Depuis 1995, la coordination du suivi de la restauration du site est confiée au Cemagref de Lyon, tandis qu'Hervé Piégay (CNRS, Laboratoire EVS) et Claude Amoros assurent le suivi géomorphologique et écologique des lônes. À partir de 2000, ce suivi particulier est intégré au suivi global de la restauration du Rhône coordonné par l'Irstea de

14 Il est aussi, sous le pseudonyme du «professeur MolloMollo ", un rédacteur régulier du journal militant La gueule ouverte (Micoud, 2001).

15 D'après les termes de la lettre au préfet de la région RhôneAlpes (juillet 1998) pour la mise en œuvre d'un plan décennal de restauration hydraulique et écologique du Rhône.
Lyon (avec Jean-Michel Olivier du LEHF et Nicolas Lamouroux de l'Irstea).

\section{Des chercheurs en sciences humaines très présents eux aussi}

Comment les sciences sociales interviennent-elles sur ce site de Vernaison ? À partir de 1998, dans le cadre de son programme scientifique, en tant qu'ethnopôle reconnu par le ministère de la Culture, l'association Maison du fleuve Rhône a mené des enquêtes sur le site de Vernaison, notamment à cause des enjeux sociaux sous-jacents du processus de restauration. Pour le dire rapidement, allait-on, sur cette friche naturelle à deux pas de la ville, « mettre la nature en ordre » pour la « nettoyer » d'une autre «faune sauvage » indésirable parce que composée de diverses activités sociales marginales, voire délictueuses (prostitution, jeux d'argent, dépotoirs...) ? (Armani et Vincent, 1999 ; Vincent, 2000).

Profitant de l'inauguration du site restauré en 2000, le directeur de l'Agence de l'eau, conscient de l'importance de la dimension sociale de ce type d'opération, s'adresse à la Maison du fleuve Rhône pour lui demander de travailler à l'édification d'une méthode de suivi socioéconomique des effets d'une restauration hydraulique et écologique susceptible d'être appliquée à tous les autres sites d'ores et déjà programmés et pour laquelle le site de Vernaison acquiert le titre de "site pilote » (Armani, 2003). En effet, en juillet 1998, les trois ministères (de l'Économie, des Finances et de l'Industrie ; de l'Équipement, des Transports et du Logement ; de l'Aménagement du Territoire et de l'Environnement) avaient chargé le préfet de la région Rhône-Alpes de mettre en œuvre un plan de restauration hydraulique et écologique du Rhône, dit ensuite plan décennal. Cette demande qui, comme on l'a vu, débute par le rappel de l'abandon du canal Rhin-Rhône et qui note également les profondes perturbations du milieu naturel causées par cet aménagement du fleuve par la CNR, cite l'opération de Vernaison en exemple pour promouvoir une restauration des débits réservés permettant de retrouver un fleuve « vif et courant » et de se rapprocher par là d'une meilleure qualité écologique.

Revenons au site de Vernaison où la Maison du fleuve Rhône commence son travail en 2003. Celui-ci est l'occasion de faire la connaissance de Geneviève Gandy, présidente dynamique du Syndicat du Haut-Rhône qui vient d'être créé et qui est membre du Comité de pilotage de l'étude menée sur Vernaison. Quand, en 2004, la Maison est choisie pour réaliser le suivi socioéconomique des effets de la restauration sur le site du Haut-Rhône, les liens, déjà noués avec la présidente du Syndicat du HautRhône, renforcent la pertinence d'une présence des 
sciences sociales en accompagnement des travaux de restauration. Notons ici ce fait d'importance qu'il est maintenant question d'un site du Haut-Rhône, consécutif à la création du Syndicat du Haut-Rhône et, tout aussi significatif, que ce nouveau syndicat prend la suite de l'ainsi nommé Syndicat intercommunal de protection des berges et bordures du Rhône en Savoie (SIPBBRS); ce qui n'existait en Savoie que pour " protéger les berges et les bordures ", s'est transmué, rejoint par les syndicats de l'Ain et de l'Isère, en un territoire dont le Haut-Rhône devient le nom.

Ainsi, en adoptant une approche différente de celle des « sciences dures", les chercheurs en sciences sociales s'impliquent aussi dans la réflexion sur le devenir du fleuve. Accompagnant nombre de projets depuis une quinzaine d'années, la Maison du fleuve Rhône est indéniablement bien insérée dans le réseau des acteurs. Elle est membre de la Zone atelier bassin du Rhône (ZABR), qui regroupe 14 laboratoires de recherche sous l'égide du CNRS, où elle rencontre les spécialistes géographes, écologues et biologistes. Les chercheurs des sciences de la vie de la ZABR, notamment $C$. Amoros et J.-P. Bravard, sont régulièrement invités à venir y présenter leurs travaux. Elle anime par ailleurs un « Réseau Rhône ", qui rassemble annuellement pour des journées ou des voyages d'étude, la grande majorité des structures culturelles, patrimoniales et touristiques situées le long du fleuve. Cette activité constitue une scène de rencontre avec les élus des nombreuses collectivités riveraines liées au Réseau. Enfin, impliquée plus récemment sur les volets culture, patrimoine et tourisme du «Plan Rhône », elle dispose par là d'une forme de présence et d'audience certaine auprès de la quasi-totalité des acteurs concernés par le fleuve ${ }^{16}$.

Sur le fond, historiquement riches de la réalité technique d'un Rhône aménagé, les investissements cognitifs des hydrobiologistes, d'une part, et des sociologues, $\mathrm{d}$ 'autre part, vont contribuer à faire venir au jour deux nouveaux fleuves Rhône: l'un, écologique, et l'autre, support de développement local patrimonial. Pour les hydrobiologistes, le territoire du Haut-Rhône est d'abord considéré comme un hydrosystème dont il convient de mieux connaître le fonctionnement pour en tirer des enseignements exportables en termes de gestion. Pour les sociologues, au contraire, quoique fortement articulé au fleuve qui le traverse, ce territoire singulier est avant tout une construction politique anthropocentrée. À son sujet, ils se demandent plutôt comment une action, écologique au départ, a été très vite transformée - la politique française de gestion concertée aidant - en un support pour le développement local d'un « petit pays » des confins.

\footnotetext{
16 Faute de subventions suffisantes, la Maison du fleuve Rhône a été mise en liquidation judiciaire en avril 2014.
}

\section{Quand le Rhône déborde ou le Rhône des riverains}

Durant l'été 1998, le préfet de la région Rhône-Alpes et la direction régionale de l'Environnement (Diren) mettent en place le Comité de pilotage du programme décennal. Y figurent au titre des collectivités territoriales le syndicat mixte qui encadre le site pilote de Vernaison depuis 1987 et le Syndicat intercommunal de protection des berges et bordures du Rhône en Savoie (SIPBBRS).

La présence de ce dernier dans le Comité de pilotage du programme s'explique par le fait que, pour les raisons déjà évoquées, ce site du Haut-Rhône a figuré très tôt dans la liste des sites prioritaires. Il faut dire qu'il est un des deux seuls sites, avec celui de Vernaison, où existe une structure locale à même de pouvoir répondre à l'invitation à la concertation.

En effet, très peu de temps après la crue de 1990, le maire de l'une des communes sinistrées avait pris l'initiative de réunir un collectif de onze communes savoyardes riveraines du Rhône sous le nom SIPBBRS, aussi appelé le Syndicat de Savoie. Sous la présidence de G. Gandy, cet organisme s'interroge sur la marche à suivre afin d'éviter les conséquences dommageables d'une nouvelle crue. L'idée d'évaluer les impacts que les aménagements du Rhône ont pu engendrer commence à naître. Pourtant, unis par la même volonté de connaître les caractéristiques du fleuve, les deux autres syndicats de l'Ain et de l'Isère rejoignent le SIPBBRS qui s'interroge désormais sur des problématiques plus globales que la simple gestion des inondations. Ces syndicats mutualisent leurs compétences en termes d'environnement, d'usages du Rhône, de paysages et d'enjeux constitués par le territoire du Haut-Rhône et lancent alors une étude, dite "l'étude bleue", afin de mieux connaître et de comprendre le fonctionnement global du fleuve. Celle-ci est confiée à trois cabinets privés et donne lieu à un rapport diffusé en 1994. En 1996, paraissent les résultats d'une autre étude préliminaire rétablissant toutes les hypothèses d'ouverture des débits réservés qui pourraient être entreprises par la Compagnie nationale du Rhône sans que cela perturbe la production électrique ${ }^{17}$. Cette étude servira par la suite de référence dans les décisions qui seront prises au sujet de ces débits réservés.

\footnotetext{
17 Le débit réservé est le débit minimal obligatoire que le gestionnaire d'un ouvrage hydraulique (barrage, centrale, seuil) doit conserver dans le lit naturel du cours d'eau entre la prise d'eau et l'aval de l'ouvrage afin de garantir la vie, la circulation et la reproduction des espèces qui vivent dans ces eaux (article L214-18 du Code de l'environnement, loi sur l'eau et les milieux aquatiques du 30 décembre 2006). Ce débit s'exprime en pourcentage du débit annuel moyen.
} 
En 1999, le collectif composé des trois mêmes syndicats s'avance encore un peu en faisant réaliser par le même ensemble de cabinets un «avant-projet technique » sur le territoire du Haut-Rhône. Publié sous le titre «Examen des conditions de réhabilitation du Vieux Rhône de Chautagne, Belley et Brégnier-Cordon", ce rapport ${ }^{18}$ reprend l'ensemble des études réalisées antérieurement et précède de très peu les différents avantprojets techniques de la restauration hydraulique et écologique proprement dite. On y retrouve les locutions caractéristiques devenues incontournables de "retour à un fleuve vif et courant » et de "restitution d'un espace de liberté ». En bref, douze années d'études hydrauliques et environnementales s'écoulent entre la crue, la création du Syndicat du Haut-Rhône le 17 avril 2003 et la signature du programme de réhabilitation hydraulique et écologique du Haut-Rhône le 20 mai 2003.

Ainsi, suite à une crue qui touche les communes des trois départements de l'Ain, de la Savoie et de l'Isère - alors même qu'elles pouvaient espérer en être protégées par les derniers aménagements de la $\mathrm{CNR}$-, les élus regroupés dans une même structure, et informés par des décennies de travaux scientifiques invitant à reconsidérer le fleuve comme un objet fluctuant et vivant, donnent au territoire de leurs 28 communes le nom de cette section du Rhône qui, morphologiquement parlant, est bien plus étendue. Alors que l'appellation «Haut-Rhône » désignait auparavant la portion du fleuve allant de la frontière suisse à Lyon, elle qualifie à présent le syndicat regroupant les communes sur lesquelles va être mis en œuvre le programme de restauration hydraulique et écologique du Haut-Rhône. Auparavant désignation hydromorphologique (et administrativo-technique, pour la CNR), elle est devenue celle d'un « territoire de projet » porté par les élus locaux. Ainsi, la réunion des collectivités riveraines du fleuve que les limites départementales avaient toujours rendue impensable, s'effectue autour du projet d'une réappropriation du Rhône, ainsi mobilisé comme levier de développement pour ce territoire proprement inventé pour l'occasion. Et, comme le terme de «restauration » y invite, il est manifeste que le programme de développement local qui s'y adosse ne peut le faire qu'en mettant fortement en scène cette nouvelle «nature » du Rhône, beaucoup plus polysémique que celle des spécialistes des sciences de la vie et de la Terre.

Mais les choses peuvent aussi être dites autrement. Depuis plusieurs décennies, le Haut-Rhône, construit de façon à la fois scientifique et militante, comme un objet hydrobiologique remarquable, a conquis les sphères administratives de la gestion de l'eau. Â ce titre, il a été choisi pour un programme emblématique de restauration hydraulique et écologique de ses bras morts et de ses

\footnotetext{
18 Il a été réalisé par trois bureaux d'études (GAY, CERREP et SOGREA).
}

sections court-circuitées. Sur la scène locale structurée par les réseaux d'élus constitués à la suite d'une crue, le fleuve ainsi reconsidéré s'offre alors comme un axe structurant le territoire qui le longe, jusqu'à lui donner son identité, et cela par-delà les découpages politicoadministratifs. Reste que, si cette restauration se voit confiée aux élus - qui ainsi "récupèrent " un fleuve -, elle doit bien se faire en concertation avec la CNR qui reste l'opérateur principal du fleuve.

Comme on peut d'ores et déjà le prévoir, les trois logiques coprésentes sur ce même lieu ne vont pas manquer de provoquer leur lot de tensions, voire de conflits. Pour les chercheurs des sciences de la vie et de la Terre tout d'abord, engagés à pérenniser les protocoles d'observation seuls à même de permettre d'évaluer scientifiquement les effets de la restauration et d'en tirer des publications, le Syndicat du Haut-Rhône qui en est le maître d'ouvrage devrait gérer son territoire un peu comme s'il s'agissait d'une réserve naturelle, quitte pour cela à devoir imposer ses vues à la CNR. Sauf que les contraintes de la CNR, opérateur des travaux de restauration, sont ailleurs : elles lui commandent de turbiner l'eau du fleuve davantage en fonction du prix du kilowatt-heure que pour des strictes prescriptions écologiques. Et c'est sans doute faute de pouvoir intervenir au cœur de cette opposition entre deux conceptions antagonistes (écologique versus économique) que le Syndicat du Haut-Rhône, troisième acteur de cette scène, s'évertue à ne pas s'y laisser enfermer en construisant de son côté un troisième fleuve : le fleuve patrimoine. La Maison du fleuve Rhône, chargée du suivi socioéconomique des effets du programme de restauration, et de fait, accompagnatrice des efforts du syndicat du Haut-Rhône pour donner consistance à son territoire, peut à ce stade être considérée comme ayant contribué à ce positionnement. C'est que, pour avoir compris tout l'intérêt qu'il pouvait y avoir à se saisir du programme de restauration, le syndicat du Haut-Rhône ne souhaitait pas pour autant y être totalement inféodé. Ce fut tout l'art de la présidente de cet ensemble de communes des confins, dont les liens avec le fleuve pouvaient être assez divers, de faire en sorte que chacune, d'une manière ou d'une autre, puisse s'en sentir solidaire.

\section{Conclusion}

En révélant le rôle des années de recherches en hydrobiologie et en écologie sur la transformation des référentiels d'action des élus, la recherche participe donc en partie de la sociologie des sciences. Mais parce qu'elle a pointé également celui des "entrepreneurs écologiques » (et «sociologiques »), elle incite à ne pas négliger la part que les actions militantes et citoyennes détiennent dans ces changements de représentation. On 
peut dire que, de ce fait, une "communauté hybride » (Callon et al., 2001) du Haut-Rhône s'est peu à peu constituée dont les membres partagent peu ou prou les mêmes attentes quant à l'avenir du fleuve. Il en est résulté qu'alors que le Rhône faisait jusqu'à présent fonction de frontière administrative entre quatre départements - avec tout ce qu'une telle situation peut entraîner de difficultés pour ces petites régions des marges, riveraines du fleuve mais éloignées des sièges des autorités départementales -, le programme de restauration a transformé le fleuve pour en faire comme l'arête dorsale d'un territoire potentiel. C'est, à nos yeux, un des effets les plus forts et les plus évidents du programme. Quoi qu'il en soit de ses résultats avérés et palpables sur le plan hydraulique et écologique, c'est d'abord et avant tout par son existence même que ce programme, en ce qu'il a permis de manifester l'existence, à cet endroit, d'un intérêt majeur porté au fleuve par un "collectif élargi », a pu être à l'origine de cette « territorialisation » impensable.

Outre cette transformation "topologique»-d'un fleuve qui unit plutôt qu'il divise -, le programme a amené d'autres changements. Alors que la "concession » du fleuve Rhône à la CNR avait pu l'escamoter, livré qu'il était à une instance externe, aujourd'hui, parce que sa restauration concrète a été l'objet d'une intense concertation (pourquoi telle lône plutôt que telle autre, comment apprécier l'augmentation des débits réservés, etc.), il est en quelque sorte réinvesti par ses riverains. Limité au début à une étroite bande disposée le long du fleuve, le territoire du maître d'ouvrage du programme s'est progressivement développé pour finir par incorporer un grand nombre de communautés de communes (de plus en plus éloignées des rives) qu'il a fallu savoir convaincre. Parallèlement, le syndicat a également considérablement élargi ses compétences pour, dès le début, intégrer nombre $\mathrm{d}$ 'initiatives connexes sans rapport étroit avec la restauration stricto sensu mais susceptibles de densifier et de complexifier le territoire : la véloroute, les patrimoines locaux, la protection de la biodiversité, etc. Un tel travail de «territorialisation » n'a pu se faire que grâce à une gouvernance à la fois très efficace et, ceci étant une condition de cela, très à l'écoute des attentes de tous les partenaires concernés, en faisant preuve de grandes qualités diplomatiques. Ce qui invite à retenir qu'aucun nouveau territoire ne saurait voir le jour sans les capacités du politique à le doter d'un projet cohérent et à le porter sans faiblir.

\section{Références}

Amoros, C., Bravard, J.-P., 1985. L'intégration du temps dans les recherches méthodologiques appliquées à la gestion écologique des vallées fluviales: l'exemple des écosystèmes aquatiques abandonnés par les fleuves, Revue Française des Sciences de l'Eau, 4, 349-364.
Amoros, C., Petts, G.E. (Eds), 1993. Hydrosystèmes fluviaux, Paris, Masson.

Armani, G., 2003. Suivi scientifique du plan décennal de restauration hydraulique et écologique du Rhône (volet socioéconomique). Définition d'une méthode. Rapport pour le Syndicat du Haut-Rhône.

Armani, G., Vincent, A., 1999. L'île de la Table Ronde, ou la restauration d'un espace fluvial au service d'une ré-appropriation territoriale, in Patrimonialisation $d u$ vivant et structuration de l'espace. Rapport de recherche, Programme de recherche "Patrimonialisation du vivant et structuration de l'espace »1996, Agence Rhône-Alpes pour les sciences sociales et humaines.

Barthélémy, C., 2006. De la restauration écologique à la concertation: des territoires fluviaux en devenir ? Rapport de recherche, Montpellier, UMR G-Eau/Lyon, Cemagref.

Bravard, J.-P., 1985. Le Haut-Rhône français : dynamique naturelle et impacts des travaux d'aménagement d'un fleuve et de sa vallée. Thèse de doctorat de géographie, Lyon, Université Lyon 3.

Callon, M., 1986. Éléments pour une sociologie de la traduction. La domestication des coquilles Saint-Jacques dans la baie de Saint-Brieuc, L'Année sociologique, 36, 169-208.

Callon, M., Lascoumes, P., Barthes, Y., 2001. Agir dans un monde incertain. Essai sur la démocratie technique, Paris, Seuil.

Carrel, G., 2002. Prospecting for historical fish data from the Rhône river basin: a contribution to the assessment of reference conditions, Archiv für Hydrobiologie, 155, 2, 273-290.

Charvolin, F., 2003. L'invention de l'environnement en France. Chroniques anthropologiques d'une institutionnalisation, Paris, La Découverte.

Fourny, M.-C., Micoud, A., 2002. Représentations et nouvelles territorialités: à la recherche du territoire perdu, in Debarbieux, B., Vanier, M. (Eds), Ces territorialités qui se dessinent, La Tour-d'Aigues/Paris, Éditions de l'Aube/ Datar, 31-52.

Fraenkel, B., 1992. La signature. Genèse d'un signe, Paris, Gallimard.

Juget, J., Amoros, C., Gamulin, D., Reygrobellet, J.-L., Richardot, M., Richoux, P., Roux, C., 1976. Structure et fonctionnement des écosystèmes du Haut-Rhône français. II., Étude hydrologique et écologique de quelques bras morts. Premiers résultats, Bulletin d'écologie, 7, 4, 479-482.

Klingeman, P.C., Bravard, J.-P., Giuliani, Y., Olivier, J.-M., Pautou, G., 1998. Hydropower reach by-passing and dewatering impacts in gravel-bed rivers, in Klingeman P.C., Beschta, R.L., Komar, P.D, Bradley, J.B. (Eds), Gravelbed rivers in the environment, Highlands Ranch, Water Resources Publications, LLC, 313-334.

Lajarge, R., Roux, E., 2007. Ressource, projet, territoire : le travail continu des intentionnalités, in Gumuchian, $\mathrm{H}$., Pecqueur, B. (Eds), La ressource territoriale, Paris, Economica/Anthropos, 133-146.

Langumier, J., 2008. Survivre à l'inondation. Pour une ethnologie de la catastrophe, Lyon, ENS Éditions.

Michelot, J.-L., 1990. Les conflits pour l'aménagement de l'espace au confluent Ain-Rhône, Annales de géographie, 99, 555, 513-530.

Micoud, A., 1995 [nouvelle édition: 2006]. Article « Territoire », in Barreyre, J.-Y., Bouquet, B., Chantreau, A. (Eds), Dictionnaire critique de l'action sociale, Paris, Bayard. 
Micoud, A., 2000. Territoire et action publique, Vie Sociale, 5/6, 15-21.

Micoud, A., 2001. Les associations de protection de la nature et de défense de l'environnement, in Boyer, M., Herzlich, G., Maresca, B. (Eds), L'environnement, question sociale, Paris, Odile Jacob, 119-129.

Micoud, A., 2007. De l'expert-militant à l'être vivant sensible, in Cosmopolitiques, 15, numéro thématique "Esthétique et espace public », 121-134.

Micoud, A., Armani, G., Olivier, J.-M., Vincent, A., 2011. Le Haut-Rhône français : d'un fleuve restauré à la construction d'un territoire. Rapport de recherche dans le cadre du programme "Eaux et territoires", Maison du fleuve Rhône, LEHNA, Syndicat du Haut-Rhône.

Monneret, M.-C., 2001. Génissiat et le Haut-Rhône. Territorialisation, fleuve, aménagement, usages et valeurs du patrimoine. Rapport de recherche, Maison du fleuve Rhône.
Muller, P., 2003. Les politiques publiques, Paris, Presses universitaires de France.

Pagès, D., 2010. La métropole parisienne et ses récits : du projet de territoire à une possible identité narrative dialoguée?, Quaderni, 73, 9-24.

Paquot, T., 2011. Qu'est-ce qu'un «territoire » ?, Vie sociale, 2, 23-32.

Piren (Programme interdisciplinaire de recherche sur l'environnement), 1982. Cartographie polythématique appliquée à la gestion écologique des eaux. Étude d'un hydrosystème flurial : le Haut-Rhône français, Paris, CNRS Éditions.

Statzner, B., Resh, V.H., Dolédec, S. (Eds), 1994. Ecology of the upper Rhône river: a test of habitat templet theories, Freshwater Biology, special issue, 31, 3, 253-554.

Vincent, A., 2000. L'île de la Table Ronde, ou le Rhône réenchanté ?, Aestuaria, 1, numéro thématique "Marais et zones humides : cultures, sociétés et territoires », 163176.

Reçu le 23 juillet 2014. Accepté le 16 mars 2015. 